\title{
UWB Power Propagation for Bio-medical Implanted Devices
}

\author{
M. Ghafari, M. Ghavami \\ BiMEC, School of Engineering \\ London South Bank University, London, United Kingdom \\ Email: meran.g@ hotmail.co.uk, ghavamim@lsbu.ac.uk
}

\begin{abstract}
This work presents a study of ultra wideband (UWB) technology to compute the energy transmission level as an electromagnetic impulse traveling from the transmitter antenna into the human body, and reaching the receiver antenna. The consideration is based on two center frequencies at $3.5 \mathrm{GHz}$ which occupies $1 \mathrm{GHz}$ bandwidth and at $6.1 \mathrm{GHz}$ occupying $6 \mathrm{GHz}$ bandwidth. A small discone antenna with gain of $1.8 \mathrm{dBi}$ is employed as the transmitter antenna and a designed implantable patch antenna with gain of $4 \mathrm{dBi}$ is also used as the receiver antenna. The distance between two antennas is $23.6 \mathrm{~mm}$ and the tissue attenuation has been considered for two layers including skin and fat with thickness of $2 \mathrm{~mm}$ and $9.6 \mathrm{~mm}$ respectively. The computation is accomplished for one way-link UWB radar system with respect to $F C C$ regulation, UWB antenna characteristic and Biological tissue model focusing on attenuation affects. The attenuation is based on the response of the transmitted incident power to reflection, absorption and thickness of the human tissue including frequency-dependent parameters such as permittivity and conductivity. Computer simulation results demonstrate the power comparison for two center frequencies in terms of bandwidth and attenuation. Since the lower band of UWB is suitable for radiation into the human body due to the greater penetration of signals, the results indicate that despite the increasing frequency from 3.5 GHz to $6.1 \mathrm{GHz}$, there is only minor power variations at the receiver.
\end{abstract}

Index Terms-UWB, UWB Waveform, UWB Antenna, Biological Tissue, Power Density.

\section{INTRODUCTION}

Ultra wideband (UWB) communication systems have been used in diverse military and commercial applications, requiring high data rate and immune to multi-path fading. Moreover, UWB impulse radio has been a promising technology in wireless communication applications due to extremely low power dissipation, short waveforms and wide bandwidth [1], [2]. In 2002, the Federal Communication Commission (FCC) authorized unlicensed use of the spectrum from $3.1 \mathrm{GHz}$ to 10.6 GHz for commercial use including indoor applications [3]. Benefiting from short electromagnetic pulses (nanosecond) and high accuracy, UWB technology has also been used in other entities like medical imaging, localization and ground penetration radar (GPR) applications [4]. In addition, UWB systems are suitable for short-distance applications as the high efficiency is at 2.5 - 5 meters for delivering data rate of $480 \mathrm{Mbps}$; correspondingly, the data rate effectively decreases as the distance increases [5]. On the other hand, one of the fundamental challenges in UWB communication systems is to maximize the magnitude level of the impulse while maintaining the spectrum within the FCC mask. The UWB communication is based on currier-free impulse transmission, leading to substantial reduction in design complexity and size which are potential advantages in the medical applications [6].

In recent years, the importance of UWB radar systems has become more attractive in biomedical applications such as vital sign monitoring, brain and breast scan as well as implanted sensors. In fact, this is due to economical cost, implantibility, penetration ability into human body, high resolution and low emission level for safety concern, referring to as the specific absorption rate (SAR) [7 ].

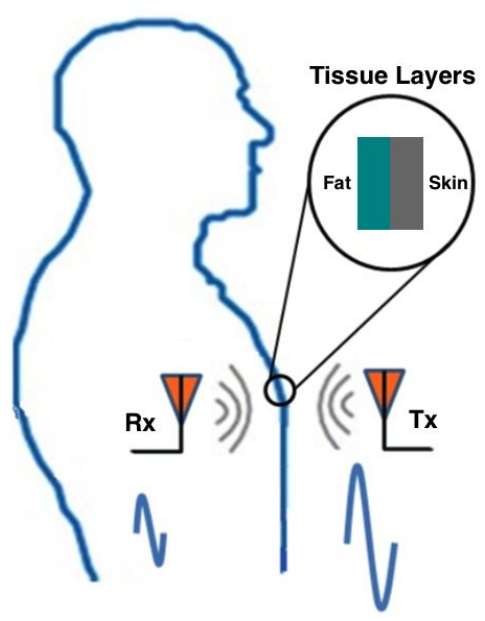

Fig. 1: Schematic representation of wireless power supply for implanted sensors using UWB.

At the same time, the feasibility of a wireless power supply (WPS) technology has been one of interesting topics in a research area with wide range of applications. One of the areas that can benefit the most is biomedical applications and in particular implanted sensors which are operating on batteries. On the contrary, there are some restrictions and challenges to transmit electromagnetic waves into the human body which can restrict the amount of transmitting and receiving power caused by the tissue's reflection and absorption. In addition, these factors are frequency-dependent and need to be considered for specifying the electromagnetic range of frequencies in order to achieve a greater power at the receiver.

This paper represents a WPS technology that is based 
on UWB radar systems in order to analyze the comparison between the power dissipations of two center frequencies at $3.5 \mathrm{GHz}$ occupying $1 \mathrm{GHz}$ bandwidth and $6.1 \mathrm{GHz}$ with 6 $\mathrm{GHz}$ bandwidth. The main focus of this work is to investigate the maximum power that can be received at the receiver antenna output for the frequency range of interest as the UWB signal travels into the human body as illustrated in Fig. 1. The simulation is based on time-domain considering the maximum transfer power according to FCC rules, tissue reflection and absorption including tissue properties such as thickness, permeability and conductivity, UWB antenna size and performance.

The remaining of this paper is organized as follows: in section II, topologies of the system is introduced. The UWB waveforms and transmitter antenna model including power density are described in sections III and IV. The biological tissue model and the receiver antenna covering power analysis are presented in sections V and VI. Simulation results are then provided in section VII and section VIII presents the concluding remarks.

\section{System Topologies}

The concept is based on a radar communication system consisting a transmitter antenna, biological tissue model and a receiver antenna as shown in Fig. 2.

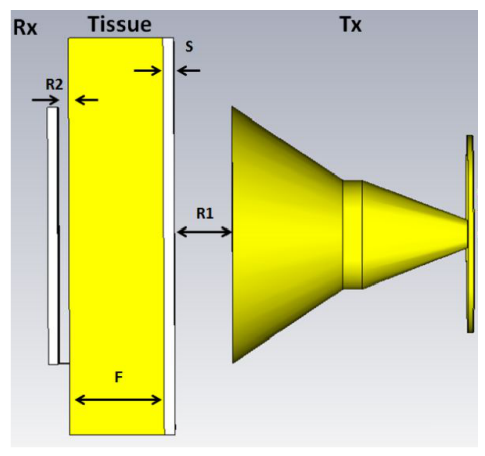

Fig. 2: Applied biological tissue between two UWB antennas.

A discone antenna is placed at distance of $\mathrm{R} 1$ form the skin surface as a transmitter radiating UWB electromagnetic waves into the biological tissue. An implantable patch antenna is placed at the distance of $\mathrm{R} 2$ from the fat layer as a receiver antenna. The distance and thickness for all elements are shown in Table I.

TABLE I: The Optimized Parameters.

\begin{tabular}{ccc}
\hline \hline Parameters & Values & Units \\
\hline Fat Thickness (F) & 9.6 & $\mathrm{~mm}$ \\
Skin thickness (S) & 2 & $\mathrm{~mm}$ \\
Tx to Skin Distance (R1) & 10 & $\mathrm{~mm}$ \\
Rx to Fat Distance (R2) & 2 & $\mathrm{~mm}$ \\
\hline
\end{tabular}

\section{UWB WAVEFORMS}

UWB impulses are characterized by an extremely wide bandwidth, good resolution range and by definition have a fractional bandwidth of 0.2 or a $10 \mathrm{~dB}$ bandwidth of $500 \mathrm{MHz}$ [8].

In general, UWB impulses should not have DC components to allow effective radiation in communication with a flat spectrum over the desired bandwidth [9]. Moreover, UWB system is associated with carrier-less impulses, time-domain format, very short pulse (nanosecond). One of the most common UWB impulses is the Gaussian pulse that its derivatives have been used in many applications due to low cost and simplicity to generate. Fig. 3 shows the derivatives of Gaussian pulse within the FCC mask.

The basic Gaussian waveform [10] has the form of:

$$
x(t)=\frac{A}{\sqrt{2 \pi} \sigma} e^{-\frac{T p^{2}}{2 \sigma^{2}}}
$$

where $\mathrm{A}$ is amplitude, $\sigma$ and $T p$ are pulse shape factor and pulse width respectively.

Generally, the first derivative of the Gaussian pulse does not meet the FCC emission requirements. One possibility is to shift the cut-off frequency higher and adjust the bandwidth by sinusoid pulse modulation which improves the manageability of the pulse in terms of cut-off frequency and bandwidth. However, since the Gaussian pulse is relatively easy to generate at low cost using analog components, the modulation will increase the cost and complexity of the transmitter. Thus, the second and fifth derivatives of Gaussian pulse have been used in this work for two center frequencies of $3.5 \mathrm{GHz}$ and $6.1 \mathrm{GHz}$ with a bandwidth of $1 \mathrm{GHz}$ and $6 \mathrm{GHz}$, respectively. Choosing the cut-off frequency of $3.5 \mathrm{GHz}$ was based on lower frequency offers better propagation characteristics and signal penetration for applications that require operation through human tissue, and the cut-off frequency of $6.1 \mathrm{GHz}$ was chosen for greater power and wider bandwidth.

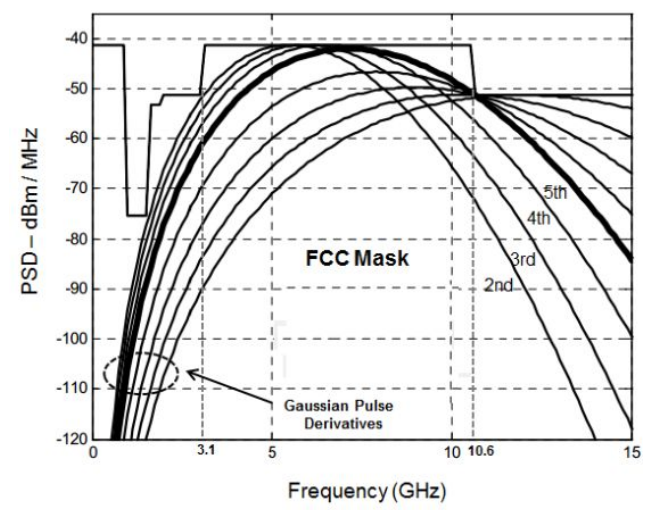

Fig. 3: UWB Gaussian pulse derivatives in frequency-domain.

\section{Transmitter Antenna Model And Power DENSITY}

Focusing on a transmitter antenna for the body area network (BAN) applications, an antenna needs to have a compact size and good performance in terms of radiation, gain, beamwidth and directivity. Several antennas (Patch, Loop, Bowtie) have been designed and simulated, however, the radiation 
efficiency reduced significantly as antenna is placed in close proximity to the skin surface $(10-15 \mathrm{~cm}$ distance) due to high reflection. As the result, we propose to use the UWB discone antenna from [11] according to its good radiation and directivity in response to the skin reflection and because it operates at lower-band of the UWB frequency range. In addition, the antenna has a wide bandwidth, small dimension ( $26 \mathrm{~mm}$ for largest length) and suitable for UWB biomedical applications. Originally, the discone antenna operates at cutoff frequency of $3.5 \mathrm{GHz}$, offering $1 \mathrm{GHz}$ of bandwidth from $3.1 \mathrm{GHz}$ to $4.1 \mathrm{GHz}$ and a gain of $1.8 \mathrm{dBi}$. For comparison we have applied some adjustments in order to shift the cut-off frequency from $3.5 \mathrm{GHz}$ to $6.1 \mathrm{GHz}$ achieving a bandwidth of $6 \mathrm{GHz}$ with a gain of $1.9 \mathrm{dBi}$.

Referring to transmitter antenna characteristics and UWB waveform bandwidth, the effective isotropic radiated power (EIRP) [12] is defined as:

$$
\operatorname{EIRP}[\mathrm{dBm}]=\mathrm{PSD}[\mathrm{dBm} / \mathrm{MHz}]+\mathrm{BW}[\mathrm{MHz}]
$$

which can also be written in terms of the transmitter power:

$$
P_{t}[\mathrm{dBW}]=\operatorname{EIRP}[\mathrm{dB}]-\text { Antenna Gain }[\mathrm{dBi}]
$$

PSD is the maximum allowing power spectrum density ($41.3 \mathrm{dBm} / \mathrm{MHz}$ ) according to FCC regulations. By applying the transmitter antenna gain and bandwidth of $F_{c}=3.5 \mathrm{GHz}$ in equations (2) and (3), the maximum transmitted power $\left(P_{t}\right)$ is $-13.1 \mathrm{dBmW}$ and if $Z_{0}=50 \Omega$, and the maximum transmitted voltage is $49.48 \mathrm{mV}$. Similarly, by applying same procedure for $F_{c}=6.1 \mathrm{GHz}$ with corresponding bandwidth, the maximum transmitted power $\left(P_{t}\right)$ is $-5.31 \mathrm{dBm}$ and the maximum transmitted voltage is $121.47 \mathrm{mV}$. The maximum power spectrum density [13] $P_{d}$ for the transmitter can be defined from:

$$
P_{d}=\frac{P_{t} G_{a}}{4 \pi R^{2}}\left[W / m^{2}\right]
$$

where $P_{t}$ and $G_{a}$ are transmitter power and antenna gain respectively. $R$ is the distance between $T_{x}$ and $R_{x}$ in meters. Using Table I, the maximum radiated power density at $3.5 \mathrm{GHz}$ frequency is $10.24 \mathrm{dBm} / \mathrm{m}^{2}$ and for $6.1 \mathrm{GHz}$ frequency is $61.44 \mathrm{dBmW} / \mathrm{m}^{2}$ which shows a significant improvement.

\section{Biological Tissue Model}

Human tissue has several layers and each one affects the RF waveform propagation independently due to its permeability, conductivity, permittivity and thickness. In this work, the biological tissue has been investigated for two layers of skin with thickness of $2 \mathrm{~mm}$ and fat with thickness of $9.6 \mathrm{~mm}$ [14]. The tissue influences on electromagnetic waves radiation and transmitter power considered by computing the attenuation for frequency of interest $(3.5 \mathrm{GHz}$ and $6.1 \mathrm{GHz})$ as the incident power density traveling into the human tissue. The most frequently encountered factors are reflection and absorption of skin and fat layers respectively as illustrated in Fig. 4. The reflection $L_{\text {ref }}$ and absorption $L_{\text {abs }}$ constants from medium $n$ to medium $n+1$ can be calculated from:

$$
L_{\mathrm{ref}}=\frac{4 \eta_{n} \eta_{n+1}^{2}}{\eta_{n+1}\left(\eta_{n}+\eta_{n+1}\right)^{2}} e^{-2 \alpha_{n+1} z_{n}}
$$

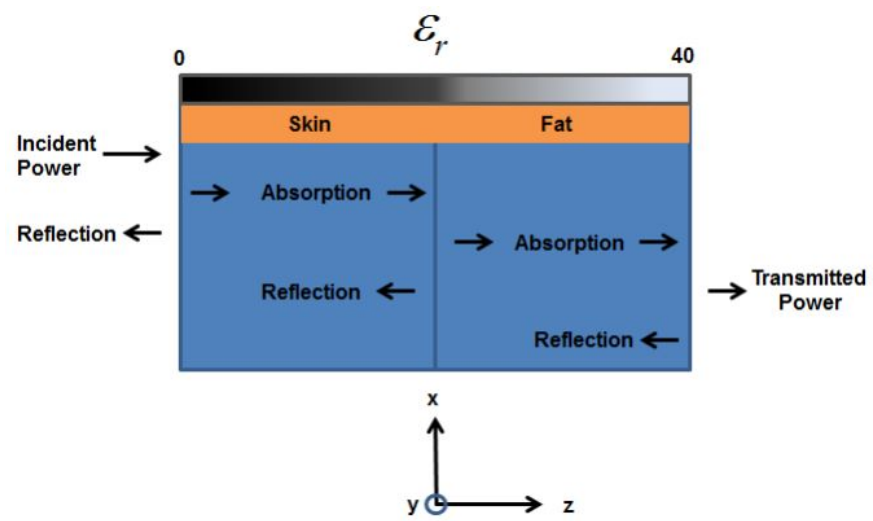

Fig. 4: Simple model of human tissue for two layers including power propagation affects into the body.

$$
L_{\mathrm{ref}}=\frac{\eta_{n}-\eta_{n+1}}{\eta_{n}+\eta_{n+1}} e^{-2 \alpha_{n+1} z_{n}}
$$

where $\eta_{n}$ and $\eta_{n+1}$ are the impedances of the first and second mediums, respectively and can be defined as:

$$
\eta=\sqrt{\frac{\mu_{0}}{\epsilon_{0} \epsilon_{r}}}[\Omega]
$$

where $\mu_{0}$ and $\epsilon_{0}$ are the permeability and permittivity of free space and $\epsilon_{r}$ is the relative permittivity. Thus, the approximate attenuation constant is:

$$
\alpha=0.5 \delta \sqrt{\frac{\mu_{0}}{\epsilon_{0} \epsilon_{r}}}\left[m^{-1}\right]
$$

where $\delta$ is the conductivity of the medium [s/m].

The attenuation has been calculated for both frequencies of $3.5 \mathrm{GHz}$ and $6.1 \mathrm{GHz}$ using equations 5 to 8 in order to determine the total received power density. The total attenuation for $3.5 \mathrm{GHz}$ frequencies range is $-9.82 \mathrm{~dB}$ (air-skin, skinfat). Subsequently, the total attenuation for $6.1 \mathrm{GHz}$ frequency range is $-15.51 \mathrm{~dB}$ from (air-skin, skin-fat). It is notable that the skin layer considered as a dry skin (non- moisturised) and the effect of blood was not taken into the account.

\section{Receiver Antenna ANd Power Analysis}

The receiver antenna structure is one of challenging parts in UWB communication especially in the case of biomedical applications. In this work, the receiver antenna needs to have a small size, good gain and directivity, low cost and be suitable for implantable applications. Focusing on requirements and various of antenna simulation results, two microstrip patch antennas have been designed and matched the requirements due to small dimension, relatively higher gain and implantability. Fig. 5 shows the position of antennas before applying biomedical tissue model in dimension of $50 \mathrm{~mm}$ by $50 \mathrm{~mm}$.

The first antenna operates at the cut-off frequency of $3.5 \mathrm{GHz}$, occupies $1 \mathrm{GHz}$ of bandwidth with gain of $3.2 \mathrm{dBi}$. Besides, the second antenna operates at cut-off frequency of $6.1 \mathrm{GHz}$ occupies $6 \mathrm{GHz}$ bandwidth with gain of $4.0 \mathrm{dBi}$. The characteristics of first antenna are shown in Table II, although, 
some adjustments are needed on the second antenna in order to achieve the cut-off frequency of $6.1 \mathrm{GHz}$.

TABLE II: Patch Antenna Parameters.

\begin{tabular}{ccc}
\hline \hline Parameters & Values & Units \\
\hline Length & 24 & $\mathrm{~mm}$ \\
Width & 17 & $\mathrm{~mm}$ \\
Substrate Thickness & 1 & $\mathrm{~mm}$ \\
Copper Thickness & 0.035 & $\mathrm{~mm}$ \\
Impedance & 50 & $\mathrm{ohm}$ \\
\hline
\end{tabular}

The return loss of $-10 \mathrm{~dB}$ obtained for the frequency range of $3.1 \mathrm{GHz}$ to $4.1 \mathrm{GHz}$ and VSWR of better than 2.0 achieved for the first antenna. Similar optimization was taken for the second antenna at frequency range of $3.1 \mathrm{GHz}$ to $9.1 \mathrm{GHz}$.

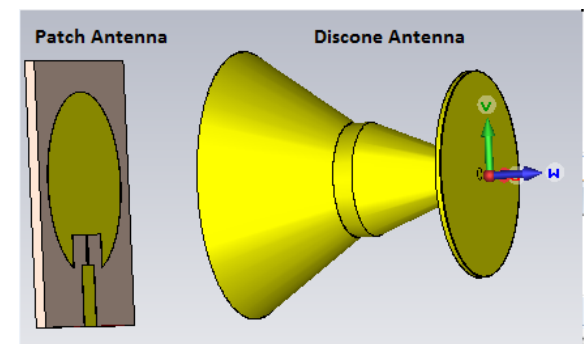

Fig. 5: Antennas communication position before applying tissue model.

Therefore, knowing the transmitter power density, biological tissue attenuation and the receiver antenna characteristics, the receiver power density can be defined as:

$$
P_{R d}=P_{d} A_{e}
$$

The effective antenna aperture [11] $\left(A_{e}\right)$ is given by:

$$
A_{e}=\frac{G_{r} \lambda^{2}}{4 \pi}
$$

where $G_{r}$ is the receiver antenna gain and $\lambda$ is wavelength.

The total received power can be calculated from equation (9) by applying equation (10). We can rewrite it in logarithmic format $(\mathrm{dB})$ for both frequencies as:

$$
\begin{aligned}
\operatorname{Pr}_{3.5}= & \mathrm{EIRP}_{\mathrm{dB}}+4+27.5-20 \log (3500) \\
& -20 \log (0.023)-9.82
\end{aligned}
$$

and

$$
\begin{aligned}
\operatorname{Pr}_{6.1}= & \mathrm{EIRP}_{\mathrm{dB}}+4+27.5-20 \log (6100) \\
& -20 \log (0.023)-15.51
\end{aligned}
$$

In this computing, we assumed that RCS components are unity and SMA ports are $50 \Omega$.

\section{Computer Simulation Results}

The computing work is setup to verify a precise range of frequencies in order to transfer maximum power through biological tissue using UWB radar system for WPS. The attenuation of human tissue has be calculated and obtained using simulation results are shown in Fig. 6. The simulation indicates that the attenuation of tissue at frequency of $3.5 \mathrm{GHz}$ and $6.1 \mathrm{GHz}$ are $-9.82 \mathrm{~dB}$ and $-15.51 \mathrm{~dB}$, respectively. In comparison, we can observe that while frequency increases, the attenuation of skin layer is quite significant; however, the attenuation for fat layer has a minor effects on both center frequencies. In fact, by increasing the center frequency, there is a $-5.7 \mathrm{~dB}$ increase of tissue attenuation which has significant lost effect on incident power. In this work, the simulation and modeling were exhibited into the time-domain using the Computer Simulation Technology (CST) and MATLAB softwares.

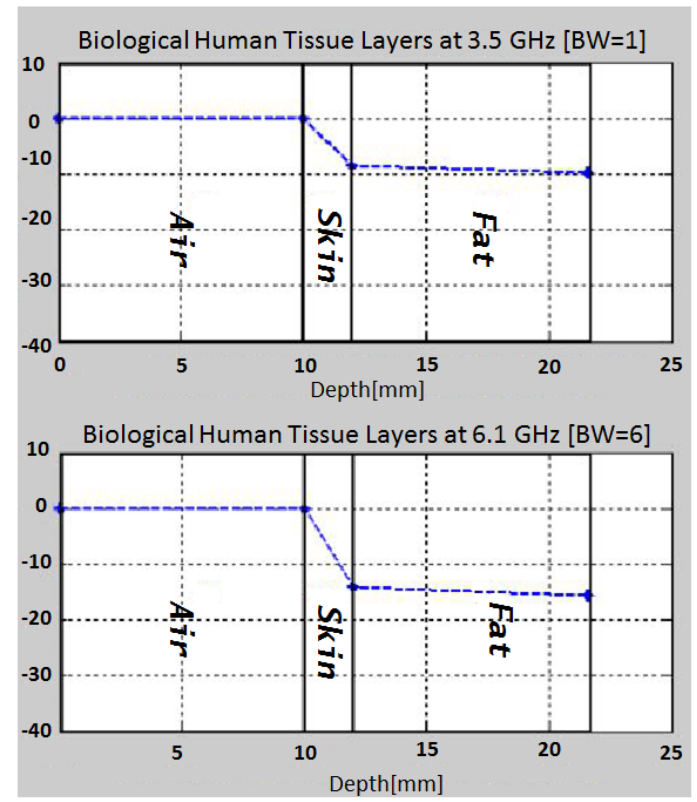

Fig. 6: Attenuation results for two layers of tissue model.

The wave propagation has been investigated for frequency range of $3.5 \mathrm{GHz}$ with a bandwidth of $1 \mathrm{GHz}$ and $6.1 \mathrm{GHz}$ with a bandwidth of $6 \mathrm{GHz}$. Since, the lower band of UWB frequencies are suitable for radiation into the human body, the penetration of signal is reduced by effect of tissue properties as frequency increases. The power budget result for frequency range of $3.5 \mathrm{GHz}$ and $6.1 \mathrm{GHz}$ are illustrated in Table III.

TABLE III: Transmitted power at frequency of $3.5 \mathrm{GHz}$ and $6.1 \mathrm{GHz}$.

\begin{tabular}{ccc}
\hline \hline Parameters & $3.5 \mathrm{GHz}$ & $6.1 \mathrm{GHz}$ \\
\hline Bandwidth [GHz] & 1 & 6 \\
EIRP [dBm] & -11.3 & -3.51 \\
$P_{t}[\mathrm{dBm}]$ & -13.1 & -5.3 \\
$P_{d}\left[\mathrm{mw} / \mathrm{m}^{2}\right]$ & 14.47 & 88.48 \\
$\mathrm{Vt}[\mathrm{mv}]$ & 0.048 & 0.29 \\
$P_{r}[\mathrm{~dB}]$ & -123.04 & -125.83 \\
$V_{r}[\mu \mathrm{v}]$ & 4.98 & 3.61 \\
\hline
\end{tabular}

The source power for $3.5 \mathrm{GHz}$ frequency is setup to $13.1 \mathrm{dBm}$ as the maximum allowance power with respect to FCC regulation and gain of antenna. By shifting the center 
frequency to $6.1 \mathrm{GHz}$, the source power can be improved by 1.3 times due to wider bandwidth which is one of method to increase the transmitter power. Basically, the result determines that by increasing the bandwidth of the impulse from $1 \mathrm{GHz}$ to $6 \mathrm{GHz}$, the transmitted power can be improved despite FCC regulation. However, considering the attenuation loss for both frequencies and applying them in power computing, the result is opposite due to reflection and absorption affect at higher frequencies. From table III, it is clear that the maximum received voltage for both frequencies is significantly low and can be a challenging task to rectify them into a usable DC voltage, however, the received DC voltage at $3.5 \mathrm{GHz}$ is 27.5 percent higher than the received DC voltage at $6.1 \mathrm{GHz}$. In other words, the received power for both frequencies are relatively close, meaning that the change of center frequency from $3.5 \mathrm{GHz}$ to $6.1 \mathrm{GHz}$ at the transmitter has minor power change at the output of the receiver antenna.

\section{CONCLUSION}

In this paper, we have presented a computational investigation of a wireless power supply through biological tissue (skin and fat) for two frequencies using a UWB radar system. We have selected the first frequency as low as possible still in range of UWB frequency $(3.5 \mathrm{GHz})$ for better penetration and the second frequency was also chosen for better power and wider bandwidth $(6.1 \mathrm{GHz})$. The operating frequency of the transmitter and receiver antennas are matched with both frequency of interest in order to maximize the power efficiency. The maximum transmitter power were calculated for both frequencies achieving $-13.1 \mathrm{dBm}$ and $-5.3 \mathrm{dBm}$ for $3.5 \mathrm{GHz}$ ( bandwidth $=1 \mathrm{GHz}$ ) and $6.1 \mathrm{GHz}$ ( bandwidth $=6 \mathrm{GHz}$ ), respectively. The tissue modeling was based on permittivity, conductivity and thickness at the frequency of interest. Simulation results for attenuation have also been compared for both frequencies in terms of power levels. In accordance with computing and simulation results, increasing frequency has significant effect on attenuation caused by the tissue reflection and absorption. The power at the receiver antenna for both frequencies are $-97.04 \mathrm{dBm}$ and $-99.88 \mathrm{dBm}$ achieving $4.98 \mu \mathrm{V}$ and $3.61 \mu \mathrm{V}$, respectively. As the result of calculation and simulation, the transmitter power at $6.1 \mathrm{GHz}$ is about 3 times higher than at $3.5 \mathrm{GHz}$. However, the received power at $3.5 \mathrm{GHz}$ is 27.5 percent higher than at $6.1 \mathrm{GHz}$ frequency due to human tissue attenuation. Therefore, the operational frequency of the transmitter could be increased in order to achieve a wider bandwidth and higher transmitter power as the received power for both frequencies are relatively close. Alternatively, improving the gain and impedance of receiver antenna and precise receiver design could be potential options to enhance the system efficiency.

\section{REFERENCES}

[1] M. Ghavami, L. B. Michael and R. Kohno, "Ultra Wideband Signals and Systems in Communication Engineering", John Wiley and sons publication, 2004
[2] K. Y. Yazdandoost, K. Hamaguchi, "Very small UWB antenna for WBAN applications", IEEE International Conference on Medical Information and Communication Technology (ISMICT ), pp.70-73, 27-30 March 2011.

[3] B. Sanz-Izquierdo, Q. Bai,P. R. Young,J. c. Batchelor, "Compact UWB Monopole for System-on-Package application", IEEE International Conference on Innovative Wireless Power Transmission , pp.771-775, 11-15 April 2011.

[4] D. Barras, F. Ellinger, H. Jcke, "A Comparison between UltraWideband and Narrowband Transceivers", Laboratory for Electronics, Swiss Federal Institute of Technology (ETH), Zurich, Switzerland, http://www2.ife.ee.ethz.ch/case/ife/publications/ comparisonOfTransceivers.pdf.

[5] R. Aiello, A. Batral, "Ultra Wideband Systems: Technology and Applications", Newnes publication, 2006.

[6] K. Chul, S. Nooshabadi, "Design of a Tunable All-Digital UWB Pulse Generator CMOS Chip for Wireless Endoscope", IEEE Transactions on Biomedical Circuits and Systems, pp.118-124, 20 - 22 March 2010.

[7] Chun-Chih. Lo, Yu-Lin. Yang, Chi-Lin. Tsai, Chieh-Sen. Lee, ChinLung. Yang, "Novel Wireless Impulsive Power Transmission to Enhance the Conversion Efficiency for Low Input Power", IEEE International Conference on Innovative Wireless Power Transmission: Technologies, Systems, and Applications (IMWS) , pp.55-58, 12-13 May 2011.

[8] T. Mohamadi, "Working Frequency in Wireless Power Transfer for Implantable Biomedical Sensors", IEEE International Conference on Electrical Engineering and Informatics, pp. 1-5, 12-17 July 2011

[9] A. Petroff, "A Practica, High Performance Ultra-Wideband Radar Platform", IEEE International Conference on Radar, pp. 880-884, 7-11 May 201

[10] G. Asachi, "An Optimization of Gaussian UWB Pulses", International Conference on Development and Application System , pp. 156 -160, 27-29 May 2006.

[11] M. Ghafari, M. Adjrad,M. Ghavami, "A Novel UWB Discone Antenna for Biomedical Applications", IEEE International Conference on Mathematical Modelling and Computer Simulation. Manchester, United Kingdom. 19 -22 Nov 2013.

[12] C. G. Bilich, "Bio-Medical Sensing using Ultra Wideband Communications and Radar Technology ", Department of Information and Communication Technology, University of Trento: PhD Proposal , 2006, www.dit.unitn.it.

[13] W. L. Stutzman, G.A. Thiele, "Antenna Theory and Design”, John Wiley and Sons, 1981 .

[14] C. G. Bilich, "UWB Radar for Bio-Medical Sensing: Attenuation Model for wave Propagation in The Body at $4 \mathrm{GHz}$, Department of Information and Communication Technology, University of Trento, www.dit.unitn.it. 